\title{
Similar healthy osteoclast and osteoblast activity on nanocrystalline hydroxyapatite and nanoparticles of tri-calcium phosphate compared to natural bone
}

\author{
This article was published in the following Dove Press journal: \\ International Journal of Nanomedicine \\ 2 December 2014 \\ Number of times this article has been viewed
}

\author{
Adam K MacMillan' \\ Francis $\vee$ Lamberti' \\ Julia N Moulton ${ }^{2}$ \\ Benjamin M Geilich ${ }^{2}$ \\ Thomas J Webster ${ }^{2,3}$ \\ 'RTI Surgical, Alachua, FL, USA; \\ ${ }^{2}$ Department of Chemical Engineering, \\ Northeastern University, Boston, \\ MA, USA $;{ }^{3}$ Center of Excellence \\ for Advanced Materials Research, \\ King Abdulaziz University, Jeddah, \\ Saudi Arabia
}

\begin{abstract}
While there have been numerous studies to determine osteoblast (bone forming cell) functions on nanocrystalline compared to micron crystalline ceramics, there have been few studies which have examined osteoclast activity (including tartrate-resistant acid phosphatase, formation of resorption pits, size of resorption pits, and receptor activator of nuclear factor $\mathrm{\kappa B}$ [RANK]). This is despite the fact that osteoclasts are an important part of maintaining healthy bone since they resorb bone during the bone remodeling process. Moreover, while it is now well documented that bone formation is enhanced on nanoceramics compared to micron ceramics, some have pondered whether osteoblast functions (such as osteoprotegerin and RANK ligand [RANKL]) are normal (ie, non-diseased) on such materials compared to natural bone. For these reasons, the objective of the present in vitro study was to determine various functions of osteoclasts and osteoblasts on nanocrystalline and micron crystalline hydroxyapatite as well as tri-calcium phosphate materials and compare such results to cortical and cancellous bone. Results showed for the first time similar osteoclast activity (including tartrate-resistant acid phosphatase, formation of resorption pits, size of resorption pits, and RANK) and osteoblast activity (osteoprotegerin and RANKL) on nanocrystalline hydroxyapatite compared to natural bone, whereas osteoclast and osteoblast functions on micron crystalline versions of these ceramics were much different than natural bone. In this manner, this study provides additional evidence that nanocrystalline calcium phosphates can serve as suitable synthetic analogs to natural bone to improve numerous orthopedic applications. It also provides the first data of healthy osteoclast and osteoblast functions on nanocrystalline calcium phosphates compared to natural bone.
\end{abstract}

Keywords: hydroxyapatite, osteoclasts, tri-calcium phosphate, nanocrystalline, nanophase, orthopedics

\section{Introduction}

Nanotechnology has already had a great impact in orthopedics since it provides the ability to better mimic the native nano-environment of natural tissues. ${ }^{1,2}$ Nanomaterials (that is, materials with one fundamental dimension less than $100 \mathrm{~nm}$ ) can reduce implant infection, inhibit inflammation, and increase bone growth all without using pharmaceutical agents. ${ }^{3}$ With these great results, nanostructured materials are leading the way for reducing musculoskeletal implant failures and promoting long-term implant success; such data have been no more apparent than for ceramics used in orthopedics.

Since the early 1970s, ceramic components have been used as hip replacements. In these conventional implants, alumina and zirconia $\left(\mathrm{ZrO}_{2}\right)$ monoliths have been the
Correspondence: Thomas J Webster Department of Chemical Engineering, Northeastern University, 313 Snell Engineering Center, Boston, MA 02115 , USA

Tel +l 6173736586

Email th.webster@neu.edu (c) (i) (5) 2014 MacMillan et al. This work is published by Dove Medical Press Limited, and licensed under Creative Commons Attribution - Non Commercial (unported, v3.0) $\mathrm{BY}$ NC License. The full terms of the License are available at http://creativecommons.org/licenses/by-nd/3.0/. Non-commercial uses of the work are permitted without any further
permission from Dove Medical Press Limited, provided the work is properly attributed. Permissions beyond the scope of the License are administered by Dove Medical Press Limited. Information on
how to request permission may be found at http://www. dovepress com/permissions php how to request permission may be found at: http://www.dovepress.com/permissions.php 
main components. Currently, though, a variety of additional bioceramics have been developed, involving three main types: bio-inert ceramics (eg, alumina, zirconia, and carbon), less resorbable bioactive ceramics (eg, sintered hydroxyapatite [HA], bioactive glass, apatite-wollastonite glass ceramics, etc), and more resorbable bioactive ceramics (eg, $\alpha$ and $\beta$-tri-calcium phosphate $[\beta$-TCP], poorly crystalline HA, tetra-calcium phosphate $[\mathrm{TeCP}]$, etc). Serving as different components, current ceramic implants have been designed with enhanced performance. ${ }^{4}$ For example, aluminum oxide $\left(\mathrm{Al}_{2} \mathrm{O}_{3}\right)$ and $\mathrm{ZrO}_{2}$ ceramics have greater bio-inertia (inability to move spontaneously) due to increased juxtaposed tissue growth, lower wear rates and reduced osteolysis than metallic and polymeric components. ${ }^{5,6}$ Silicon carbide (SiC) and silicon nitride $\left(\mathrm{Si}_{3} \mathrm{~N}_{4}\right)$ components can also provide high mechanical hardness, strength and corrosion resistance, ${ }^{7}$ and have demonstrated a strong potential as reinforcement for bone implants.

However, clearly, the ceramics which have garnered the most attention in contemporary orthopedics are calcium phosphates. Today, there are several types of commercially available calcium phosphate products for orthopedic applications, including HA, $\beta$-TCP, and biphasic calcium phosphates (TCP/HA) to name just a few. With a similar composition to bone, $\mathrm{HA}\left(\mathrm{Ca}_{10}\left(\mathrm{PO}_{4}\right)_{6}(\mathrm{OH})_{2}\right)$ is one of the most widely used chemistries for bioactive materials. ${ }^{8}{ }^{8}$ Due to its ability to promote osteoconduction, the presence of HA could enhance long-term performance of orthopedic implants. ${ }^{10,11}$ As one of many examples, studies on HA coated stainless steel enhanced bone growth compared to uncoated steel scaffolds. ${ }^{12}$ However, pure HA typically has poor mechanical properties and as such, for its application in load bearing applications, HA has been utilized as a coating or in combination with other materials with high mechanical strength. ${ }^{13}$

Nanostructured versions of these ceramics have indeed demonstrated the ability to improve implant performance. For example, HA nanoparticle coatings on tantalum enhanced bone growth compared to uncoated tantalum scaffolds. ${ }^{14}$ Combined with metals or polymers, bioactive ceramics induce specific biological responses which promote physical or chemical bonds between implants and surrounding tissue, ${ }^{6,15,16}$ with nano versions of these ceramics doing even better than micron equivalents. Also, it has been shown that nosocomial infections on nanostructured musculoskeletal ceramic implants can be reduced. Chronic bacterial infection of an orthopedic prosthetic can serve as a septic focus leading to osteomyelitis, acute sepsis, and even death. ${ }^{17}$ Specifically, Staphylococcus epidermidis (S. epidermidis) is one of the most frequently found bacteria colonizing on orthopedic implants. ${ }^{18}$ A recent study showed that $S$. epidermidis functions were inhibited on $\mathrm{ZnO}$ and $\mathrm{TiO}_{2}$ which possessed nanoscale compared to micron surface features. ${ }^{19}$

Despite this promise in using nanoceramics as improved bone substitutes, few studies have been conducted concerning osteoclast activity on nanoceramics. ${ }^{20}$ Bone remodeling involves bone resorption by osteoclasts and synthesis by osteoblasts. It is a tightly regulated process by the receptor activator of nuclear factor $\kappa \mathrm{B}$ ligand (RANKL)/receptor activator of nuclear factor $\mathrm{\kappa B}(\mathrm{RANK}) /$ osteoprotegerin (OPG) molecular triad (Figure 1). RANKL, a member of the tissue necrosis factor (TNF) superfamily, induces osteoclast differentiation, activation, and survival upon interaction with its receptor RANK. RANKL and RANK have, thus, been linked to bone loss under pathological and physiological conditions. RANK and RANKL represent the formation and maintenance of healthy bone next to an implant. Moreover, while results of increased bone growth on nanoceramics have been widespread and now confirmed since the original study published in 1998, ${ }^{21}$ some have wondered if osteoblast responses on nanoceramics follow normal processes (ie, non-diseased states) compared to natural bone. For all of the above reasons, the objective of this study was to determine osteoclast (including tartrate-resistant acid phosphatase

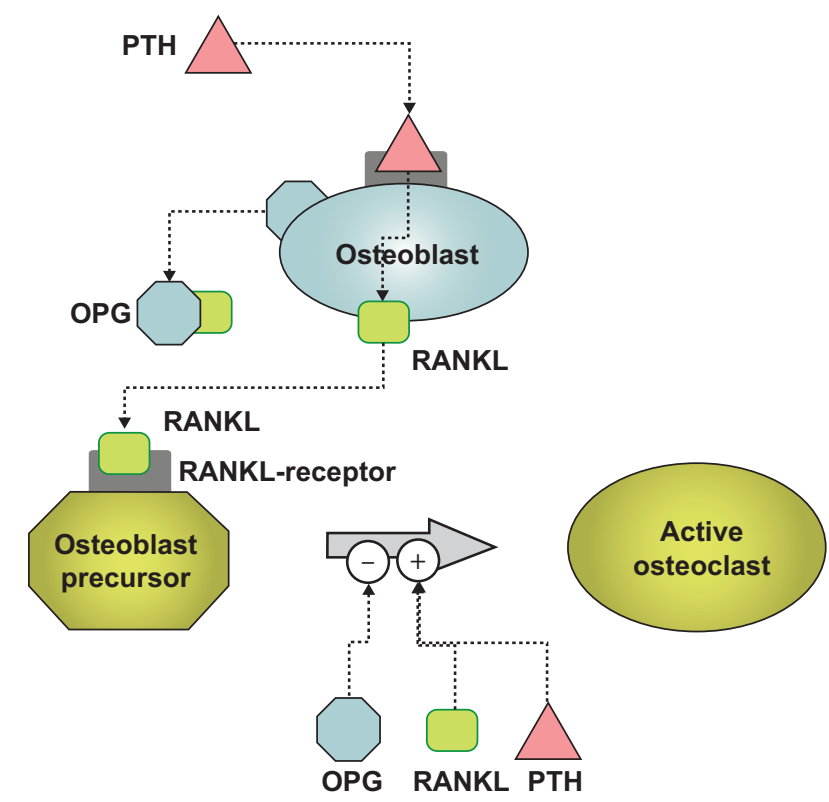

Figure I The role of RANKL, RANK, and OPG in the bone remodeling process. Notes: PTH stimulates osteoblasts to secrete RANKL which then stimulates osteoclast precursors to become active. Osteoprotegerin (OPG) is a competitive inhibitor of RANKL and thus blocks RANKL from activating osteoclasts. PTH is secreted by some forms of cancer, including breast cancer cells, and PTH is secreted by the parathyroid glands. PTH is not the only stimulator of osteoblast differentiation and this study utilized dexamethasone for similar purposes. In addition, we used vitamin D3 to induce osteoblast differentiation from bone-marrow cells as well as calcitonin to inhibit osteoclast functions.

Abbreviations: PTH, parathyroid hormone; RANK, receptor activator of nuclear factor $\kappa B$; RANKL, RANK ligand. 
(TRAP), formation of resorption pits, size of resorption pits, and RANK expression) and osteoblast (OPG and RANKL expression) functions on various types of nanoceramics and compare such responses to micron equivalents and natural bone. In this manuscript, the term "healthy" refers to a normal molecular pathway or functions of cells when compared to bone.

\section{Methods \\ Materials}

The following osteoconductive scaffolds were evaluated in the present study: i) cancellous bone, ii) cortical bone, iii) porous low crystallinity nano-HA (nanOss; RTI Surgical Corporation, Alachua, FL, USA), iv) porous low crystallinity nano- $\beta$-TCP (RTI Surgical Corporation), v) porous low crystallinity biphasic nano-TCP/HA (RTI Surgical Corporation), vi) dense sintered nano-HA (RTI Surgical Corporation), vii) porous silica-substituted micron-HA (Actifuse; Baxter Healthcare Corporation, Deerfield IL, USA), viii) porous micron-TCP (Vitoss; Stryker Corporation, Kalamazoo, MI, USA), and ix) porous biphasic micron-TCP/HA (Mastergraft; Medtronic Corporation, Minneapolis, MN, USA). Human cancellous bone obtained from the ilium and cortical bone was obtained by sectioning the tibia $(10 \mathrm{~mm} \times 10 \mathrm{~mm} \times 2 \mathrm{~mm})$ of cadaveric donors (LifeLink Tissue Bank, Tampa FL, USA). Samples were provided free of all cellular material and lyophilized. All materials tested consisted of only inorganic material and were granular in form (1-4 mm) with the exception of cortical bone and dense sintered nano-HA. Peroxide digestion was used to remove the collagen-based organic phase from the cancellous and cortical bone samples to isolate the mineral phase of the osteoconductive scaffolds. Dense sintered nano-HA was prepared by pressure-assisted sintering of nano-HA to create $10 \mathrm{~mm} \times 2 \mathrm{~mm}$ diameter discs with effectively zero porosity.

Porous silica-substituted micron-HA, porous micronTCP, and porous, micron-biphasic TCP/HA were each obtained from the above stated commercial suppliers and were provided in a sterile "ready to use" condition. All samples tested contained the calcium phosphate mineral phase only.

\section{Material characterization}

Surface area of the materials of interest to the present study was measured by multipoint Brauner, Emmit, and Teller (BET) (TriStar 3000; Micromeritics, Norcross, GA, USA). Scanning electron microscopy (SEM) (Hitachi S-4800; Hitachi Ltd., Tokyo, Japan) was used to evaluate the grain size of the materials. Images were taken at magnifications ranging from $7,000 \times$ to $40,000 \times$ as required. The crystalline phase of each material was confirmed via X-ray diffraction (Geigerflex model D/Max - IIB, Rigaku Americas, The Woodlands, TX, USA). Materials were analyzed in the supplied condition and after thermal treatment to $1,000^{\circ} \mathrm{C}$ in air for 60 minutes (min) to distinguish the characteristic peaks of the calcium phosphates. Representative scans were obtained covering the region of the major characteristic peaks (29-38 20).

\section{Osteoclast experiments}

Rat bone-marrow osteoclast-like cells were obtained using procedures modified from literature reports. ${ }^{20}$ All animal experiments were approved by the Animal Use and Care Committee at Northeastern University, Boston, MA, USA. Briefly, Wistar rat (2-3 week old) femurs were removed, dissected free of tissue, the ends of the tibiae cut off, and the marrow cavities flushed by slowly injecting 3-5 mL of Dulbecco's Modified Eagle's Medium (DMEM) containing 10\% fetal bovine serum (FBS) and 1\% antibiotic/antimycotic solution at one end of each femur. The isolated marrow tissue was centrifuged (at 21 $g$ at room temperature), resuspended in fresh DMEM (containing $10 \% \mathrm{FBS}, 10^{-8} \mathrm{M} 1-, 25-[\mathrm{OH}]$ vitamin $\mathrm{D}_{3}$ [Calbiotech, Spring Valley, CA, USA] and 1\% antibiotic/antimycotic solution) either in the presence or absence of $10^{-8} \mathrm{M}$ calcitonin (Sigma-Aldrich Co., St Louis, MO, USA), and seeded under standard cell culture conditions (that is, under a $37^{\circ} \mathrm{C}$, humidified, $5 \% \mathrm{CO}_{2} / 95 \%$ air environment) on the substrates mentioned above. Vitamin $\mathrm{D}_{3}$ was added to induce osteoclast differentiation from the hematopoietic cells of the bonemarrow. ${ }^{20}$ These cells were previously characterized by their multi-nucleated morphology, positive staining for TRAP, and bone resorptive activity (which was inhibited in the presence of calcitonin).$^{20}$ In this study, the synthesis of TRAP, bone resorptive activity, and total intracellular protein synthesis was determined as described in the next paragraph.

Primary osteoclast-like cells were used in the experiments and were isolated from the bone-marrow aspirates described above according to standard procedures. ${ }^{20}$ Cells were seeded ( $10^{6}$ cells) onto the substrates of interest to the present study and cultured under standard cell culture conditions in DMEM supplemented with $10 \%$ FBS, $10^{-8} \mathrm{M} 1-, 25-(\mathrm{OH})$ vitamin D (Calbiotech) and 1\% antibiotic/antimycotic solution for 7, 14, and 21 days. At the end of the prescribed time periods, adherent cells were rinsed twice with $\mathrm{Ca}$ - and $\mathrm{Mg}$-free phosphate buffered saline, and lysed with distilled water during three freeze thaw cycles. TRAP activity was then measured in each supernatant containing the lysates of cells according to established protocols. Briefly, aliquots from supernatant solutions were incubated with $8 \mathrm{mM}$ p-nitrophenylphosphate 
in $0.1 \mathrm{M}$ sodium acetate, $5 \mathrm{mM}$ ascorbic acid, $0.1 \%$ Triton $\mathrm{X}-100$ and $10 \mathrm{mM}$ sodium tartrate (pH 5.4) (all chemicals from Sigma-Aldrich Co.) at $37^{\circ} \mathrm{C}$ for $10 \mathrm{~min}$; the reaction of p-nitrophenol to p-nitrophenolate was stopped by adding $0.05 \mathrm{M} \mathrm{NaOH}$. Light absorbance of these samples was measured on a spectrophotometer (MR600 Spectrophotometric Microplate Reader; Dynatech) at $400 \mathrm{~nm}$.

Resorption pits were determined by seeding osteoclasts obtained from rat bone-marrow cells $\left(10^{8}\right)$ onto the surface of the substrates of interest to the present study and cultured under standard cell culture conditions in DMEM (containing

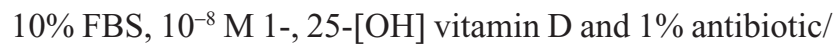
antimycotic solution) either in the absence or presence of $10^{-8} \mathrm{M}$ calcitonin for 7,10 , and 13 days. At the end of each prescribed time period, the samples were rinsed with ammonium hydroxide, sonicated, and stained with crystal violet. Resorption pits on the ceramic and bone surfaces were visualized using reflected light microscopy (Olympus I70; Olympus Corporation, Tokyo, Japan) with image analysis software (Image Pro); the number of resorption pits formed as a result of the osteolytic activity of osteoclast-like cells was counted. Resorption pit density (number of pits/cm) was determined by averaging the number of resorption pits in five random fields $\left(2 \mathrm{~mm}^{2}\right)$ per substrate. Resorption pits on substrates of interest to the present study were also visualized by SEM (Hitachi S4800; Hitachi Ltd.).

Lastly, total protein content in these cell lysates was determined spectrophotometrically using a commercially available kit (Pierce Chemical Co., Rockford, IL, USA) and by following the manufacturer's instructions. For this purpose, aliquots of each protein-containing, distilled-water supernatant were incubated with a solution of copper sulfate and bicinchoninic acid at $37^{\circ} \mathrm{C}$ for $30 \mathrm{~min}$. Light absorbance of these samples was measured on an MR600 Spectrophotometric Microplate reader at $570 \mathrm{~nm}$. Protein concentration in each of these samples was determined from standard curves of absorbance versus known concentrations of bovine serum albumin ( $5 \mathrm{mg} / \mathrm{mL}$; Sigma-Aldrich Co.) run in parallel with the experimental samples. Protein concentration was used to normalize TRAP assay results and for the mRNA analysis as described below.

\section{ELISA and RT-PCR experiments}

In this study, to ascertain normal (non-diseased) osteoclast and osteoblast functions, enzyme-linked immunosorbent assays (ELISA) were performed to quantify relative concentrations of RANK/RANKL/OPG using standard procedures. ${ }^{22}$ Specifically, RANK from osteoclasts was determined while RANKL and OPG from osteoblasts were determined. Real-time polymerase chain reaction (RT-PCR) was also used to confirm mRNA expression for RANK/ RANKL/OPG. For these assays, cells were isolated from bone-marrow aspirates as described in the last paragraph of the previous page and were culturing at 40,000 cells $/ \mathrm{cm}^{2}$ on the proposed materials for 7,14 , and 21 days in complete medium. To induce osteoblast differentiation from bonemarrow aspirates, $10^{-8} \mathrm{M}$ dexamethasone (Calbiotech) was added to DMEM supplemented with $10 \%$ FBS and $1 \%$ penicillin/streptyomycin (Hyclone, St. Louis, MO, USA). At the end of the prescribed time period, RANK, RANKL, and OPG expression was determined according to standard ELISA or RT-PCR procedures (RayBiotech, Norcross, GA, USA). ${ }^{22}$ Proteins were normalized using the assay described in this same paragraph and glyceraldehyde 3-phosphate dehydrogenase (GAPDH) was used as the housekeeping gene. Since elevated levels of RANKL have been linked to numerous bone diseases that involve bone loss, one would expect to see lower levels of RANKL on materials which promoted bone health (or levels similar to those on natural healthy bone). However, such RANKL levels will need to be high enough to stimulate some osteoclast activity for the maintenance of healthy bone.

\section{Statistical analysis}

All experiments were completed in triplicate with three repeats each. Differences were investigated within groups between time points and across groups at each time point using analysis of variance (ANOVA). Post hoc Tukey's tests were used to evaluate differences between bone, nano, and micron materials.

\section{Results and discussion}

Results of the present study confirmed the expected grain size, surface area, and crystallinity of the materials tested. Specifically, grain sizes and surface area results are shown in Table 1 while representative SEM images at 7,000 $\times$ and $40,000 \times$ are shown in Figures 2 and 3, respectively. X-ray diffraction results are shown in Figures 4 and 5. Results showed that the micron sized materials and sintered nano-HA were predominately crystalline, while nano-TCP, nano-TCP/ $\mathrm{HA}$, nano-HA, and human bone mineral were poorly crystalline. Further, human bone mineral, sintered nano-HA, nano-HA, and silica-substituted HA all exhibited an HA crystalline phase with no evidence of other material phases present. Nano-TCP/HA and micron-TCP/HA both exhibited a combination of HA and $\beta$-TCP phases. Nano-TCP and micron-TCP exhibited a $\beta$-TCP phase with no evidence of other material phases present. 
Table I The materials of interest to the present study and their grain size and surface area

\begin{tabular}{llll}
\hline Material & Grain size range $(\mathbf{n m})$ & Grain size average $(\mathbf{n m})$ & Surface $\mathbf{a r e a}\left(\mathbf{m}^{2} / \mathbf{g}\right)$ \\
\hline Cancellous bone & $25-75$ & 47 & 123.95 \\
Cortical bone & $15-115$ & 52 & 38.88 \\
Porous nano-HA & $25-75$ & 45 & 61.10 \\
Porous nano-TCP & $25-50$ & 32 & 73.60 \\
Porous biphasic nano-TCP/HA & $20-60$ & 38 & 65.11 \\
Dense sintered nano-HA & $25-140$ & 75 & 0.06 \\
Porous Si-substituted micron-HA & $700-2,600$ & 1,335 & 0.63 \\
Porous micron-TCP & $1,250-3,500$ & 2,212 & 0.35 \\
Porous micron-biphasic TCP/HA & $750-4,000$ & 1,365 & 0.33 \\
\hline
\end{tabular}

Abbreviations: HA, hydroxyapatite; TCP, tri-calcium phosphates.
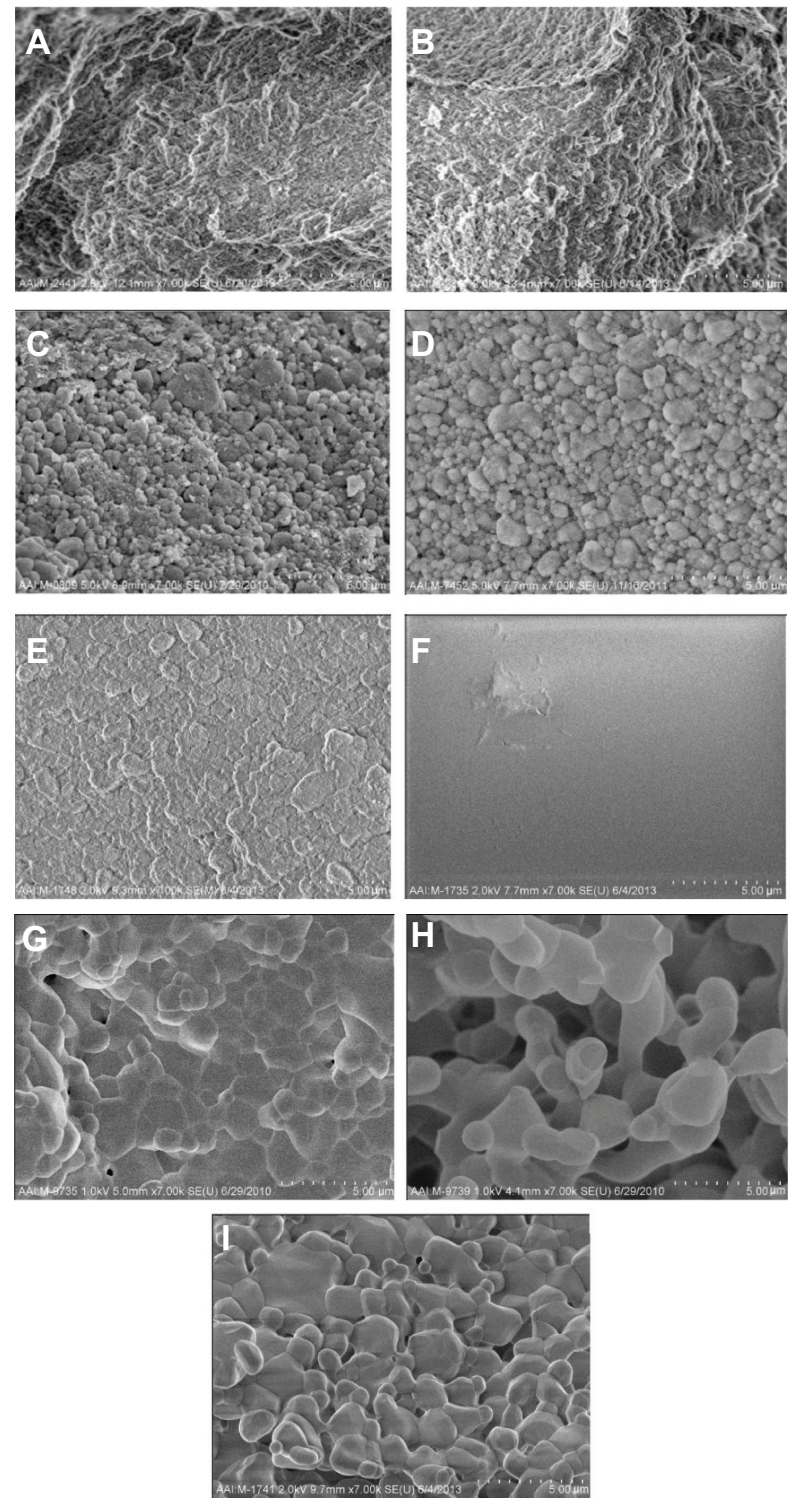

Figure 2 SEM images of all calcium phosphate samples of interest to the present study.

Notes: All images are at a magnification of 7,000 $\times(5 \mu \mathrm{m}$ scale bar). (A) Cancellous bone, (B) cortical bone, (C) porous nano-HA, (D) porous nano-TCP, (E) porous biphasic nano-TCP/HA, (F) dense sintered nano-HA, (G) porous silica-substituted micron-HA, (H) porous micron-TCP, and (I) porous biphasic micron-TCP/HA. The crystal structure of dense nano-crystalline HA cannot be seen at this low magnification.

Abbreviations: SEM, scanning electron microscopy; HA, hydroxyapatite; TCP, tricalcium phosphates.
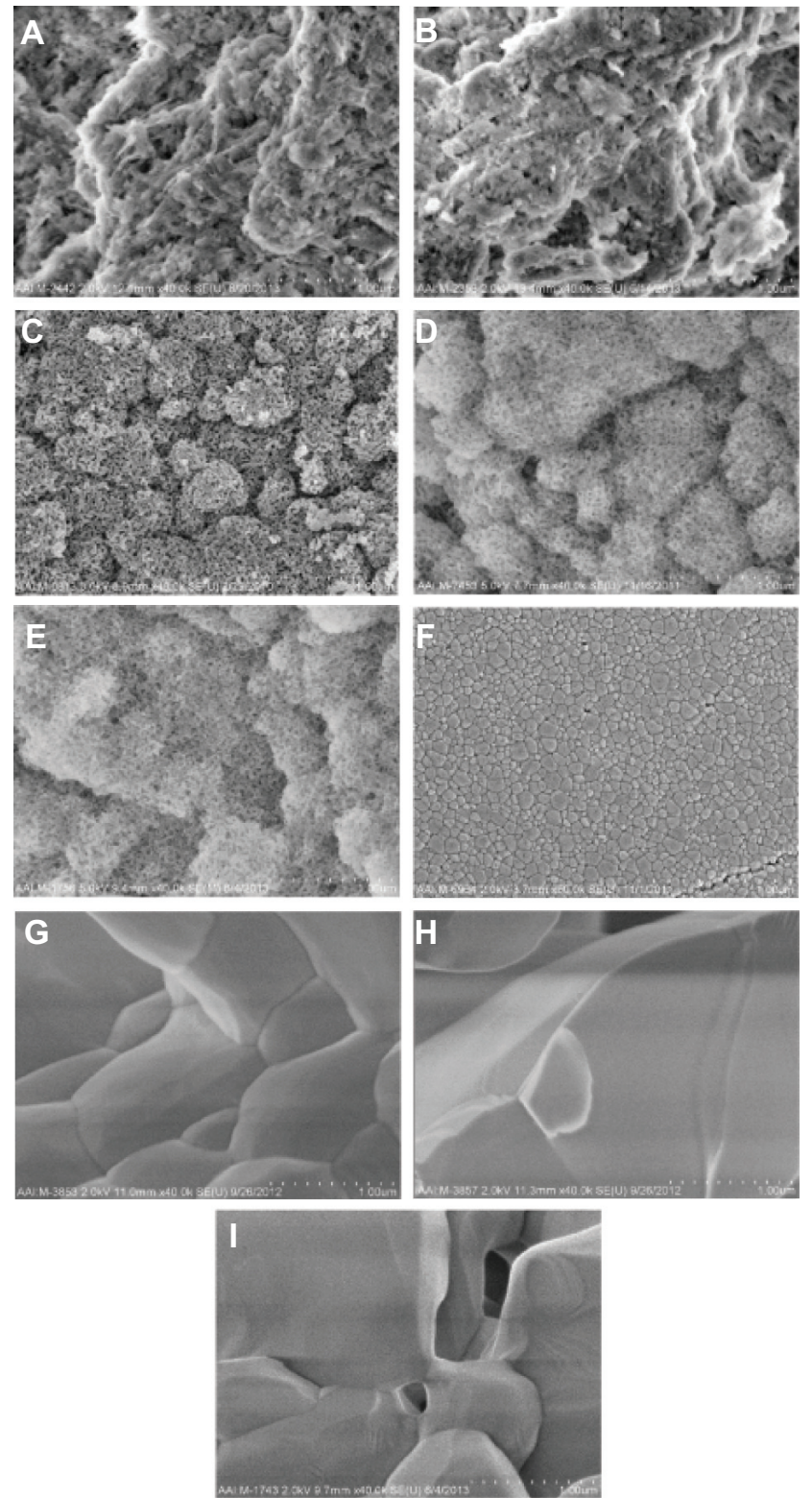

Figure 3 SEM images of all calcium phosphate samples of interest to the present study at higher magnifications.

Notes: All images are at a magnification of $40,000 \times($ I $\mu \mathrm{m}$ scale bar). (A) Cancellous bone, (B) cortical bone, (C) porous nano-HA, (D) porous nano-TCP, (E) porous biphasic nano-TCP/HA, (F) dense sintered nano-HA, (G) porous silica-substituted micron-HA, (H) porous micron-TCP, and (I) porous biphasic micron-TCP/HA.

Abbreviations: SEM, scanning electron microscopy; HA, hydroxyapatite; TCP, tricalcium phosphates. 


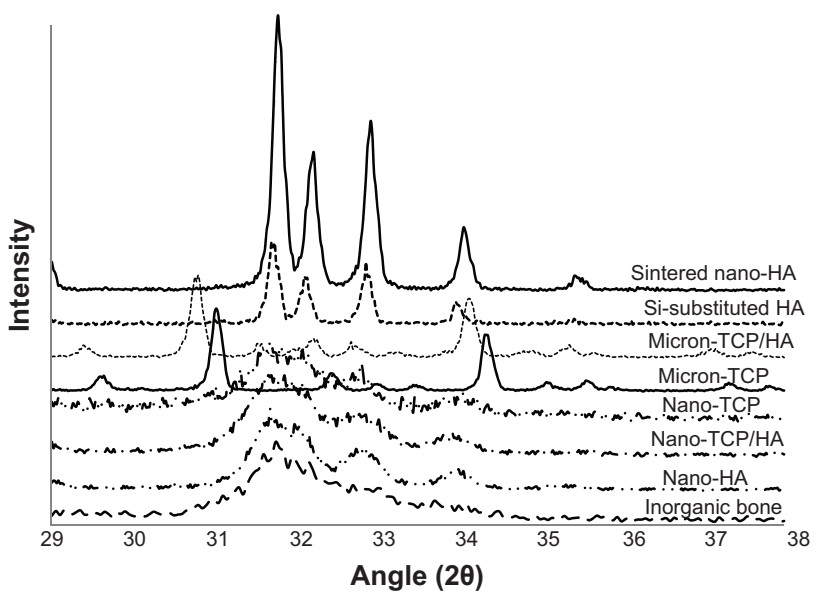

Figure $4 \mathrm{X}$-ray diffraction patterns of the calcium phosphate materials as tested. Notes: Diffraction patterns are shown on a common diffraction angle scale. It can be seen that the micron sized materials and sintered nano-HA were predominately crystalline, while nano-TCP, nano-TCP/HA, nano-HA, and human bone mineral were poorly crystalline.

Abbreviations: HA, hydroxyapatite; TCP, tri-calcium phosphates.

More importantly, results of the present in vitro study demonstrated for the first time similar TRAP activity (Figure 6) and number of resorption pits (Figure 7) formed by osteoclasts on porous nano-HA, porous nano biphasic TCP/ HA, porous nano-TCP compared to cancellous and cortical bone after each time period. In contrast, TRAP activity and number of resorption pits on the micron equivalents were not similar to either cancellous or cortical bone, with the exception of the number of resorption pits on micron-TCP

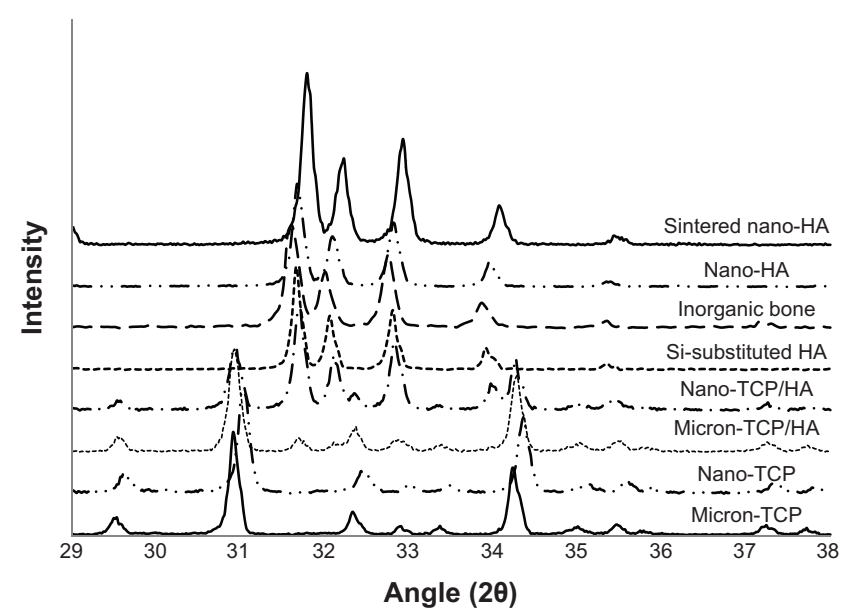

Figure $5 \mathrm{X}$-ray diffraction patterns of the calcium phosphate materials after thermal treatment at $1,000^{\circ} \mathrm{C}$ for 60 minutes.

Notes: Diffraction patterns are shown on a common diffraction angle scale depicting the characteristic peaks. Human bone mineral, sintered nano-HA, nano-HA, and silica-substituted HA all exhibited an HA crystalline phase with no evidence of other material phases present. Nano-TCP/HA and micron-TCP/HA both exhibited a combination of HA and $\beta$-TCP phases. Nano-TCP and micron-TCP exhibited a $\beta$-TCP phase with no evidence of other material phases present.

Abbreviations: HA, hydroxyapatite; TCP, tri-calcium phosphates; $\beta$-TCP, $\beta$-tricalcium phosphates. at 21 days culture. The size of the resorption pits formed by osteoclasts was similar on porous nano-HA compared to cortical bone, and porous nano-TCP, nano biphasic TCP/HA, and sintered nano-HA compared to cancellous bone after each time period (Figures 8 and 9). In contrast, the size of the resorption pits formed by osteoclasts was not similar on all other micron materials compared to bone. Importantly, these results suggest that regardless of surface area (porous versus dense), increased responses of osteoclasts were measured on nanocrystalline compared to micron crystalline calcium phosphate based materials. Moreover, similar results to cellular behavior on bone were obtained regardless of crystalline phase as long as nano compared to micron materials were compared.

Similar results were observed when comparing mRNA expression from cells on the nanomaterials compared to natural bone. Specifically, RANK/RANK/OPG mRNA expression was confirmed using RT-PCR while ELISA showed similar RANKL from osteoblasts for cancellous bone, cortical bone, porous nano-HA and dense nano-HA suggesting a more natural osteoblast to osteoclast communication on such nanomaterials compared to bone (Figure 10). In addition, similar RANK from osteoclasts for cancellous bone, cortical bone, porous nano-HA and dense nano-HA were measured (Figure 11). Lastly, similar OPG from osteoblasts for cancellous bone, cortical bone, porous nano-HA and dense nano-HA was determined (Figure 12). Such results provide additional evidence that the nanomaterials fabricated in this study promote normal osteoclast and osteoblast activity compared to natural bone, whereas, micron equivalents do not.

In this manner, the results from this in vitro study provide some of the first (if not the first) valuable information concerning normal healthy cellular processes on nanoscale materials at the molecular level. In particular, to the best of the authors' knowledge, this is the first study to report a comparable normal RANL/RANKL pathway of bone cells cultured on nano-HA to bone. Since the results here demonstrate a similar healthy molecular pathway of bone cells (both osteoblasts and osteoclasts) on nano-HA samples compared to bone, this study provides supportive data for their continued use in medicine. For example, although requiring more experiments, since altered pathways or amounts of RANK/ RANKL were not measured on nano-HA compared bone, this study begins to dispel thoughts of altered molecular pathways of bone formation on nanomaterials. Such indications of healthy bone formation and maintenance on nanomaterials demonstrate that indeed the previously observed elevated 


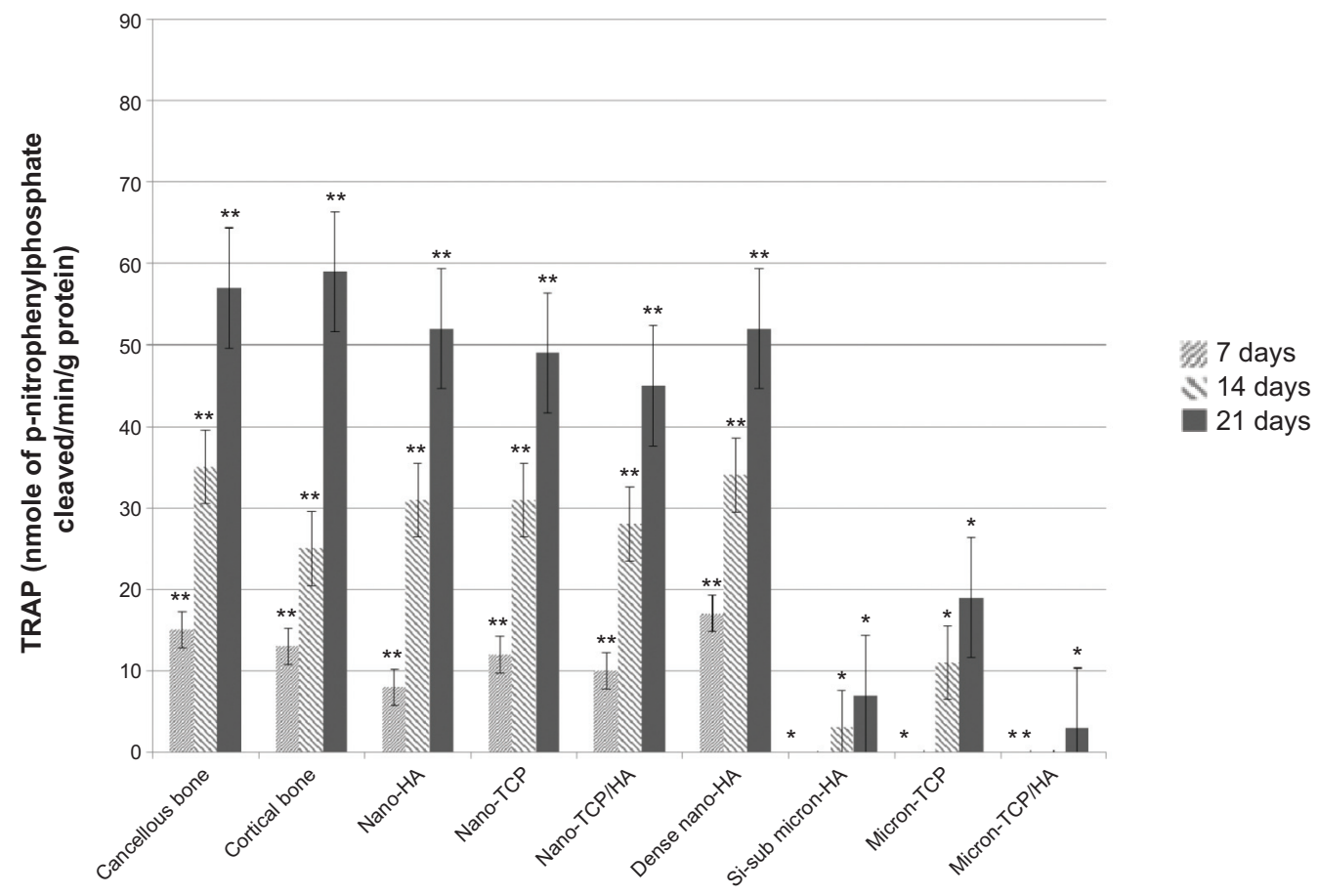

Figure 6 TRAP activity on the selected calcium phosphate materials.

Notes: TRAP activity decreased with increased crystal grain size. Data $=$ mean \pm standard error of the mean; $\mathrm{N}=3 ; * \mathrm{P}<0.0 \mathrm{I}$ compared to cancellous or cortical bone at the same time period; ** $<<0.0$ l compared to all micron materials ( $\mathrm{HA}, \mathrm{TCP}$, and TCP/HA) at the same time period. All values are significantly different at subsequent time periods for all nano and bone samples.

Abbreviations: HA, hydroxyapatite; Si-sub, Si-substituted; TCP, tri-calcium phosphates; TRAP, tartrate-resistant acid phosphatase.

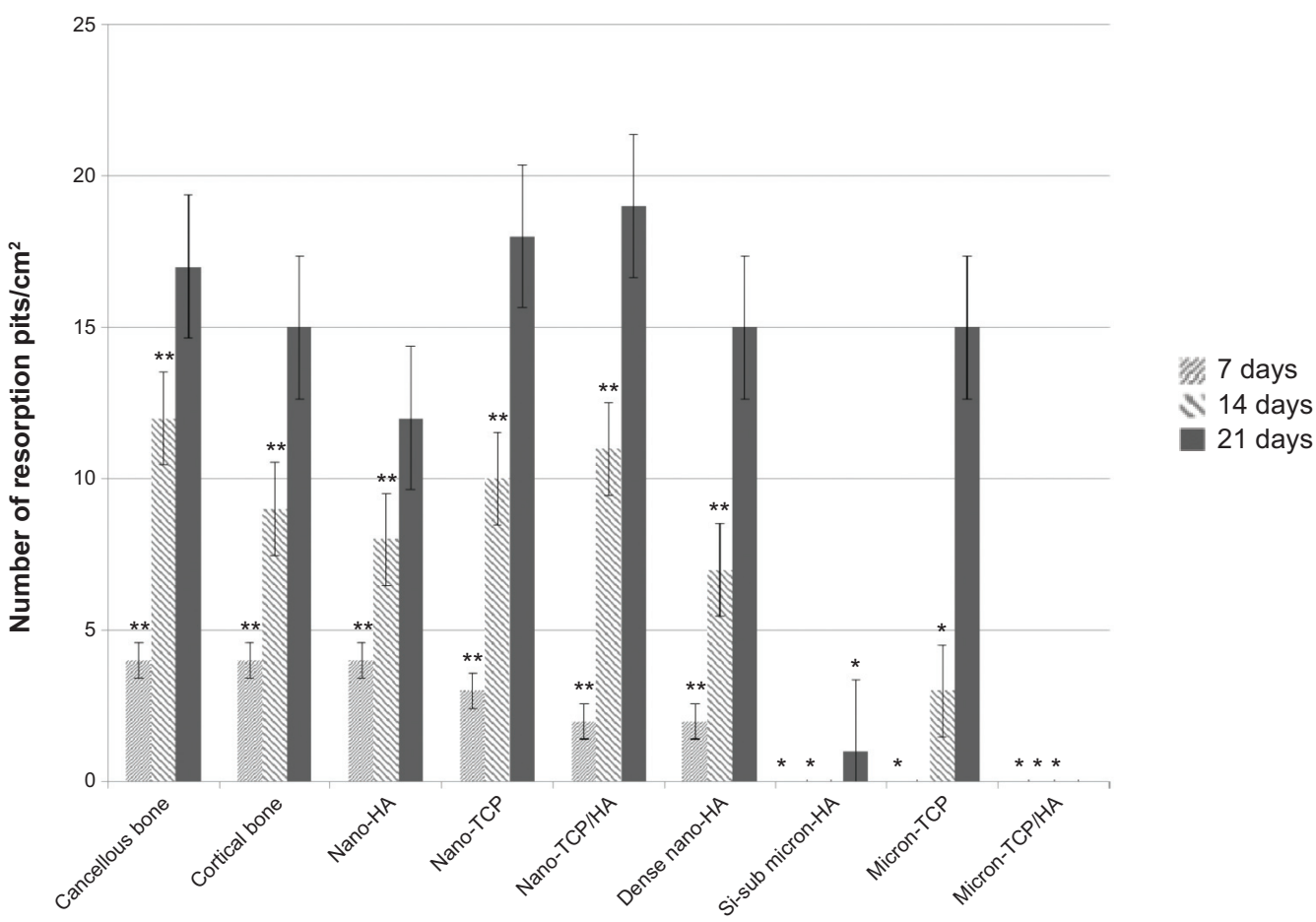

Figure 7 Number of resorption pits on the selected calcium phosphate materials.

Notes: The number of pits per unit area decreased with increased crystal grain size. Data $=$ mean \pm standard error of the mean; $N=3 ; * P<0.0$ I compared to cancellous or cortical bone at the same time period; ${ }^{*} * P<0.01$ compared to all micron materials $(\mathrm{HA}, \mathrm{TCP}$, and TCP/HA) at the same time period. All values are significantly different from one time period to the next for the same sample (except Si-substituted micron-HA and micron HA/TCP).

Abbreviations: HA, hydroxyapatite; Si-sub, Si-substituted; TCP, tri-calcium phosphates. 


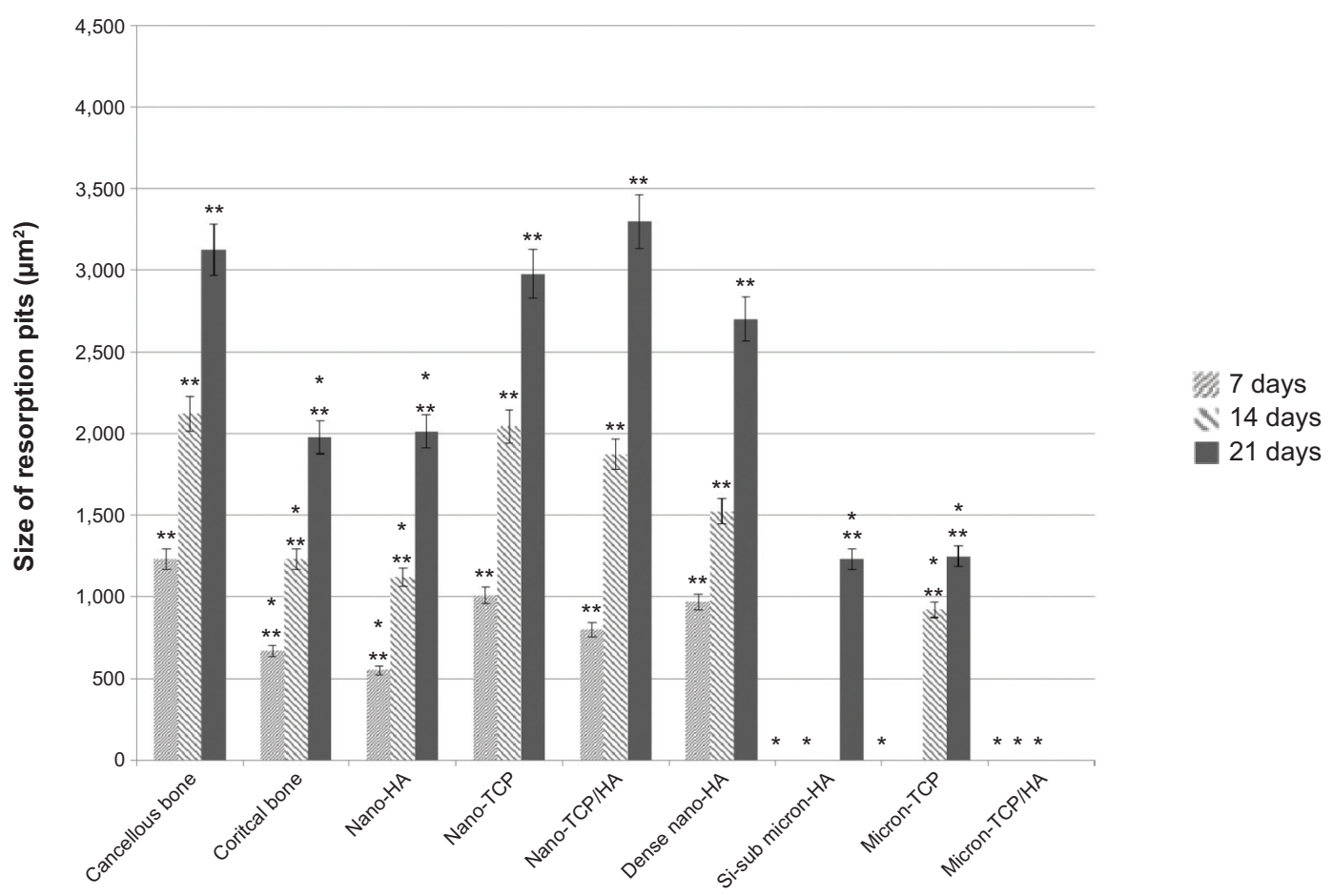

Figure 8 Size of resorption pits on the selected calcium phosphate materials.

Notes: The size of resorption pits decreased with increased crystal grain size. Data $=$ mean \pm standard error of the mean; $\mathrm{N}=3$; $* \mathrm{P}<0.0 \mathrm{I}$ compared to cancellous bone at the same time period; $* * P<0.01$ compared to all micron materials ( $\mathrm{HA}, \mathrm{TCP}$, and TCP/HA) at the same time period. All values are significantly different from one time period to the next for the same sample (except for Si-substituted micron-HA and micro HA/TCP).

Abbreviations: HA, hydroxyapatite; Si-sub, Si-substituted; TCP, tri-calcium phosphates.

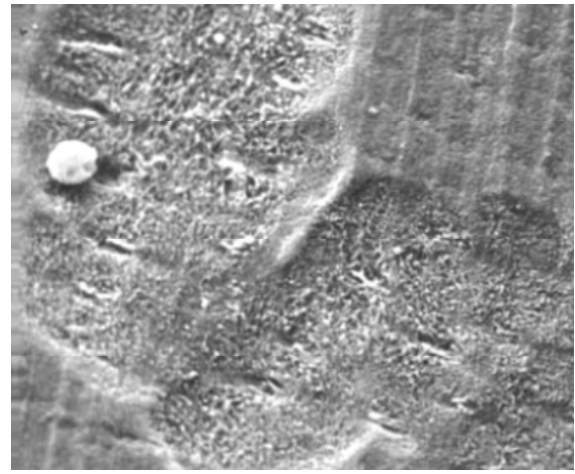

Cancellous bone

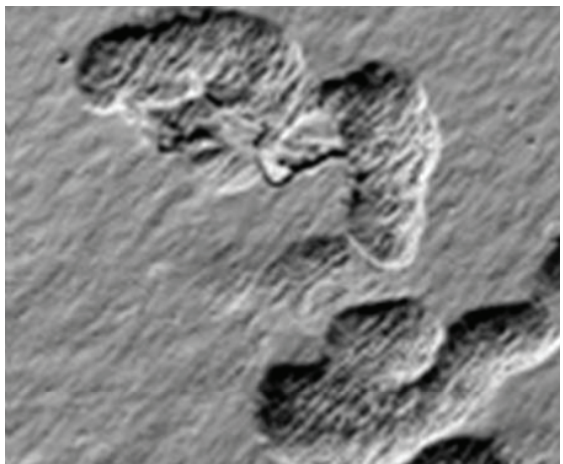

Porous nanOss ${ }^{\circledR}$ Bioactive 3D

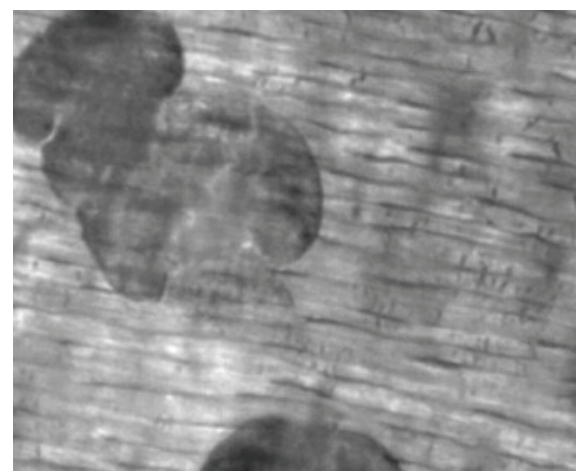

Cortical bone

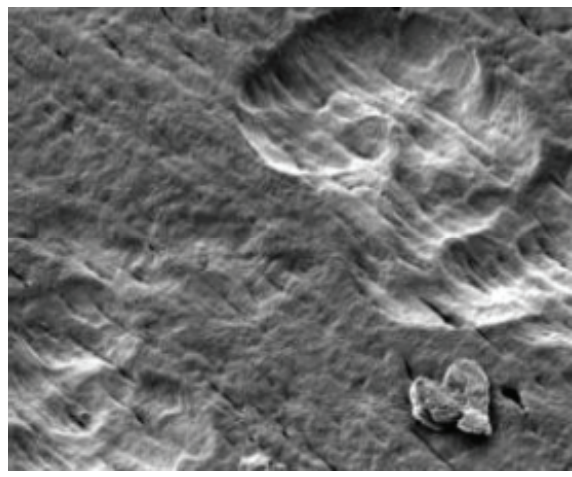

Porous nano-TCP

Figure 9 (Continued) 


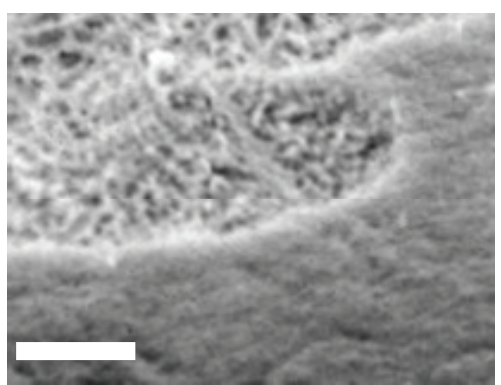

Porous nano-HA/TCP

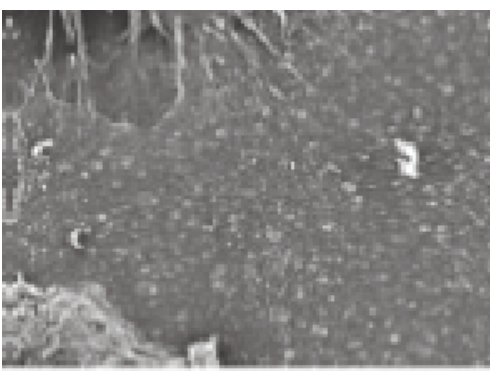

Porous micro-TCP

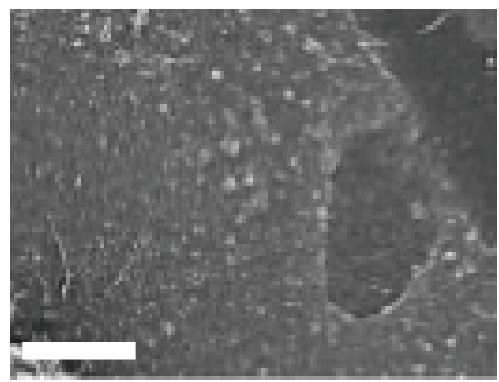

Dense nano-HA

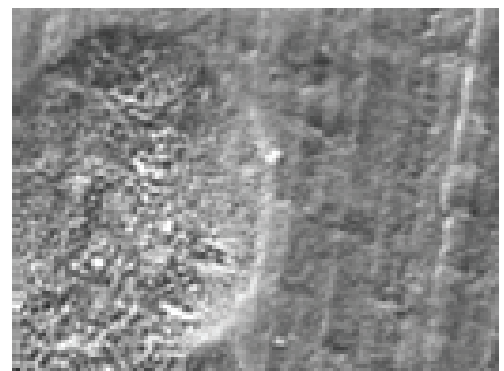

Porous micro-HA

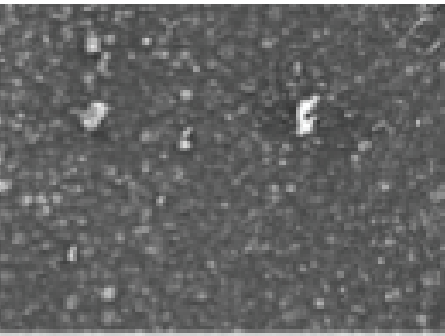

Porous micro-HA/TCP

Figure 9 Representative SEM images of osteoclast resorption pits on the various substrates after 21 days of culture. Note: Magnifications $=10,000 \times$.

Abbreviations: SEM, scanning electron microscopy; HA, hydroxyapatite; TCP, tri-calcium phosphates.

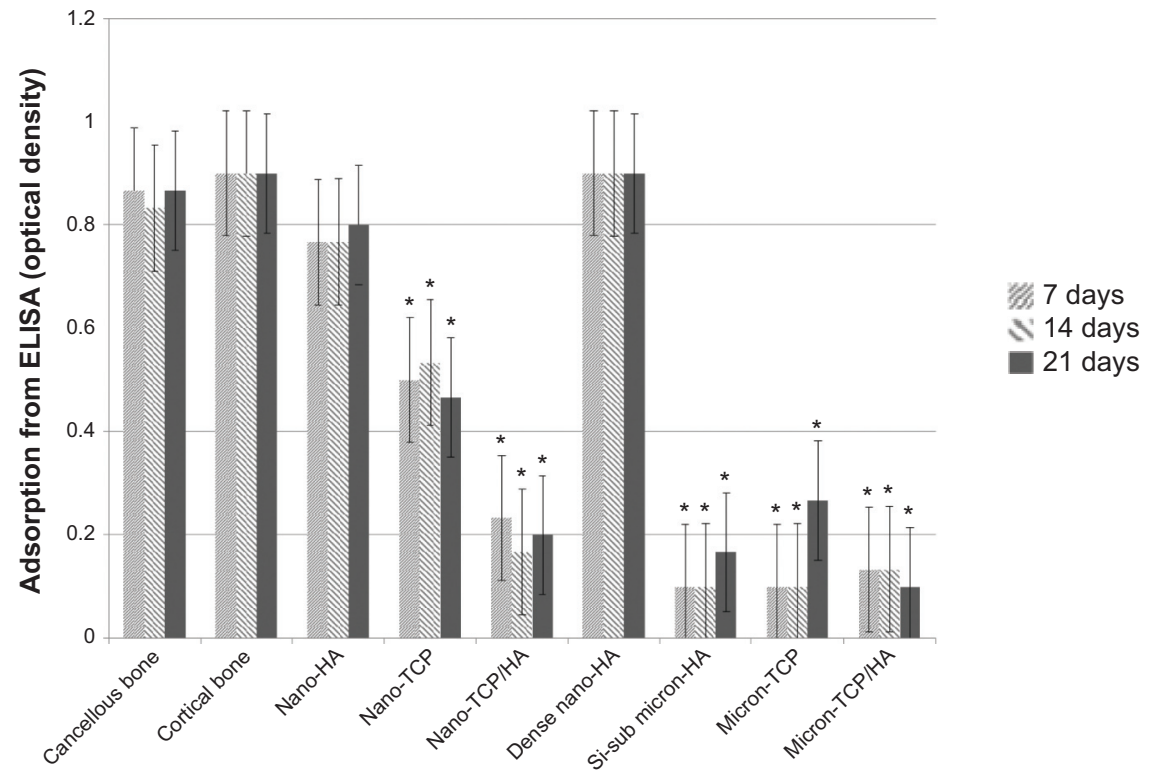

Figure 10 Similar RANKL from osteoblasts for cancellous bone, cortical bone, porous nano-HA and dense nano-HA.

Notes: $\mathrm{Data}=$ mean \pm standard error of the mean; $\mathrm{N}=3 ; * \mathrm{P}<0.0 \mathrm{I}$ compared to cancellous bone at the same time period. All values for cancellous bone, cortical bone, porous nano-HA, and dense nano-HA are significantly different than the rest at all respective time periods.

Abbreviations: HA, hydroxyapatite; TCP, tri-calcium phosphates; ELISA, enzyme-linked immunosorbent assays; Si-sub, Si-substituted; RANK, receptor activator of nuclear factor $\kappa B$; RANKL, RANK ligand. 


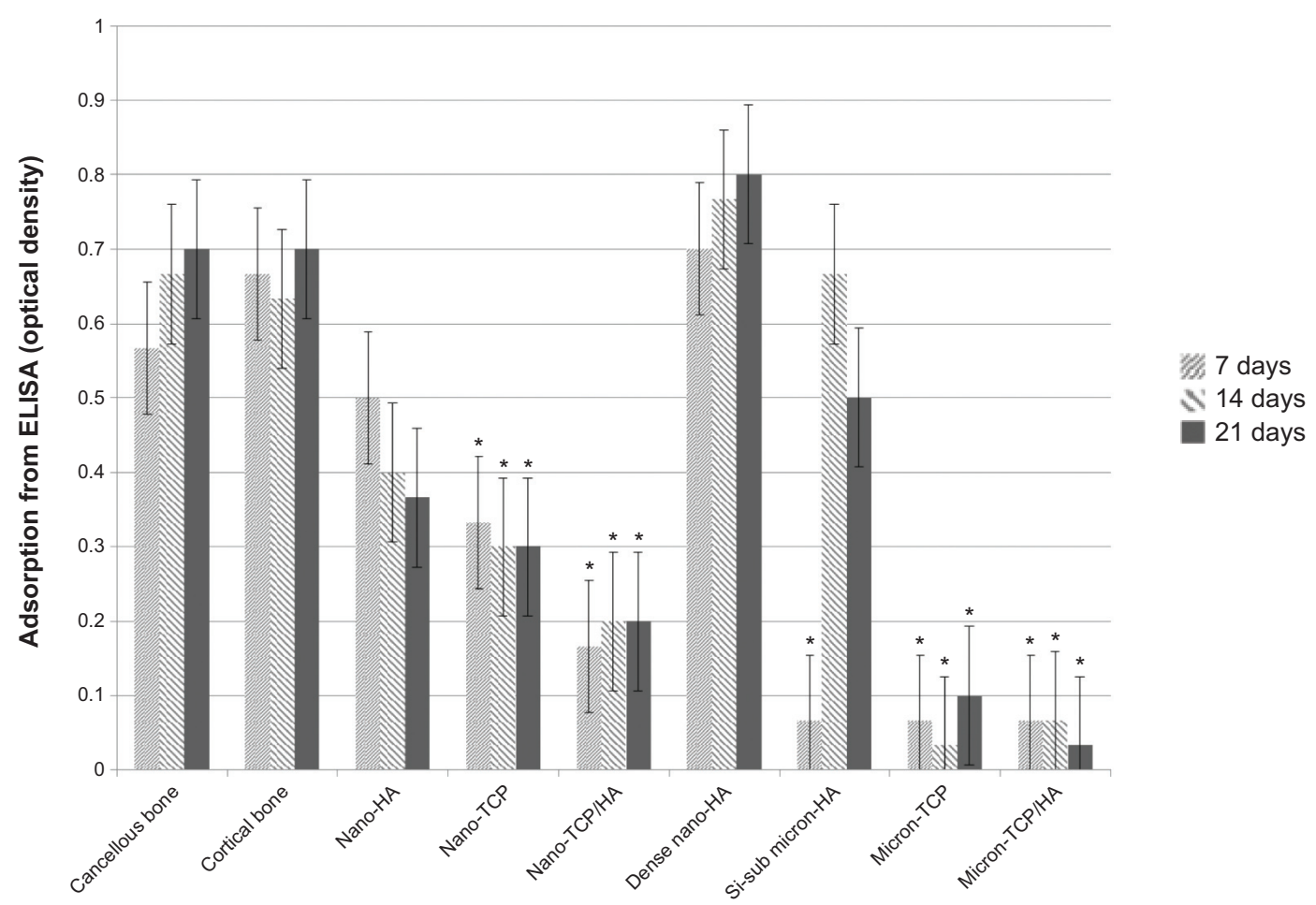

Figure II Similar RANK from osteoclasts for cancellous bone, cortical bone, porous nano-HA and dense nano-HA.

Notes: $\mathrm{Data}=$ mean \pm standard error of the mean; $\mathrm{N}=3 ; * \mathrm{P}<0.0 \mathrm{I}$ compared to cancellous bone at the same time period. All values for cancellous bone, cortical bone, porous nano-HA, and dense nano-HA are significantly different than the rest at all respective time periods.

Abbreviations: HA, hydroxyapatite; TCP, tri-calcium phosphates; ELISA, enzyme-linked immunosorbent assays; Si-sub, Si-substituted; RANK, receptor activator of nuclear factor $\kappa \mathrm{B}$.

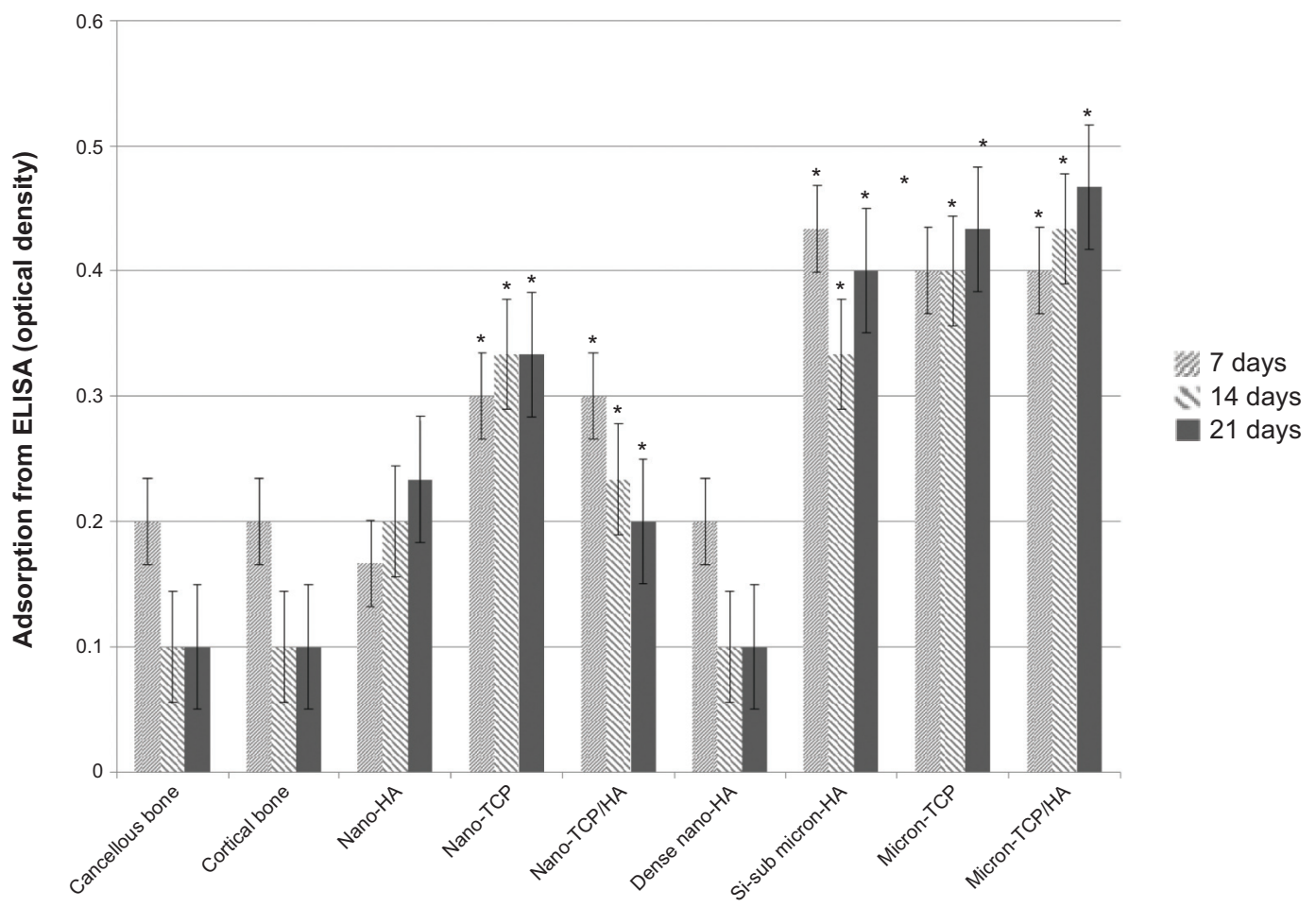

Figure 12 Similar OPG from osteoblasts for cancellous bone, cortical bone, porous nano-HA and dense nano-HA.

Notes: Data $=$ mean \pm standard error of the mean; $N=3 ; * P<0.0$ I compared to cancellous bone at the same time period. All values for cancellous bone, cortical bone, porous nano-HA, and dense nano-HA are significantly different than the rest at all respective time periods.

Abbreviations: HA, hydroxyapatite; TCP, tri-calcium phosphates; ELISA, enzyme-linked immunosorbent assays; OPG, osteoprotegerin; Si-sub, Si-substituted. 
healthy cellular processes occurring on nanomaterials are normal, just elevated..$^{15,20,21}$

\section{Conclusion}

Results of the present study showed for the first time similar osteoclast activity (including TRAP, formation of resorption pits, size of resorption pits, and RANK) and osteoblast activity (OPG and RANKL) on nanocrystalline HA compared to human bone mineral, whereas osteoclast and osteoblast functions on micron crystalline versions of these ceramics were much different than natural bone. In this manner, this study provides additional evidence that nanocrystalline HA and nanoparticulate TCP can serve as suitable synthetic analogs to natural bone to improve numerous orthopedic applications. It also provides the first data of healthy osteoclast and osteoblast functions on nanocrystalline calcium phosphates compared to bone and other micron crystalline calcium phosphate materials being used in orthopedics today.

\section{Acknowledgment}

The authors would like to thank RTI Surgical for funding the proposed study.

\section{Disclosure}

The authors report no conflicts of interest in this work.

\section{References}

1. Dvir T, Timko BP, Brigham MD, Naik SR, Karajanagi SS, Levy O, et al. Nanowired three-dimensional cardiac patches. Nat Nanotechnol. 2011;6(11):720-725.

2. Harrison BS, Atala A. Carbon nanotube applications for tissue engineering. Biomaterials. 2007;28(2):344-353.

3. Taylor E, Webster TJ. Reducing infections through nanotechnology and nanoparticles. Int J Nanomedicine. 2011;6:1463-1473.

4. Gerhardt LC, Boccaccini AR. Bioactive Glass and Glass-Ceramic Scaffolds for Bone Tissue Engineering. Materials. 2010;3(7):3867-3910.

5. Guazzato M, Albakry M, Ringer SP, Swain MV. Strength, fracture toughness and microstructure of a selection of all-ceramic materials. Part II. Zirconia-based dental ceramics. Dent Mater. 2004;20(5):449-456.
6. Chevalier J. What future for zirconia as a biomaterial? Biomaterials. 2006;27(4):535-543.

7. Sternitzke M. Structural ceramic nanocomposites. J Eur Ceram Soc. 1997;17(9):1061-1082.

8. Ginebra MP, Traykova T, Planell JA. Calcium phosphate cements as bone drug delivery systems: A review. J Control Release. 2006;113(2): $102-110$.

9. LeGeros RZ. Properties of osteoconductive biomaterials: Calcium phosphates. Clin Orthop Relat Res. 2002;(395):81-98.

10. Jarcho M, Bolen CH, Thomas MB, Bobick J, Kay JF, Doremus RH. Hydroxyapatite synthesis and characterization in dense polycrystalline form. J Mater Sci. 1976;11(11):2027-2035.

11. Dorozhkin SV. Nanosized and nanocrystalline calcium orthophosphates. Acta Biomater. 2010;6(3):715-734.

12. Ballarre J, Lopez DA, Cavalieri AL. Frictional and adhesive behavior of organic-inorganic hybrid coatings on surgical grade stainless steel using nano-scratching technique. Wear. 2009;266(11-12):1165-1170.

13. Hong YL, Fan HS, Li B, Guo B, Liu M, Zhang XD. Fabrication, biological effects, and medical applications of calcium phosphate nanoceramics. Mater Sci Eng R-Rep. 2010;70(3-6):225-242.

14. Sato M. Nanophase hydroxyapatite coatings for dental and orthopedic applications [theses]. West Lafayette: Purdue University; 2006.

15. Dulgar-Tulloch AJ, Bizios R, Siegel RW. Human mesenchymal stem cell adhesion and proliferation in response to ceramic chemistry and nanoscale topography. J Biomed Mater Res Part A. 2009; 90A(2):586-594.

16. Barone DT, Raquez JM, Dubois P. Bone-guided regeneration: from inert biomaterials to bioactive polymer (nano) composites. Polym $A d v$ Technol. 2011;22(5):463-475.

17. Cramton SE, Gerke C, Götz F. In vitro methods to study staphylococcal biofilm formation. In: Ron JD, editor. Methods in Enzymology. Academic Press; 2001:239-255.

18. Arciola CR, Campoccia D, An YH, et al. Prevalence and antibiotic resistance of 15 minor staphylococcal species colonizing orthopedic implants. Int J Artif Organs. 2006;29(4):395-401.

19. Colon G, Ward BC, Webster TJ. Increased osteoblast and decreased Staphylococcus epidermidis functions on nanophase $\mathrm{ZnO}$ and $\mathrm{TiO}_{2}$. J Biomed Mat Res Part A. 2006;78A(3):595-604.

20. Webster TJ, Siegel RW, Bizios R. Enhanced functions of osteoclast-like cells on nanophase ceramics. Biomaterials. 2001;22(11):1327-1333.

21. Webster TJ, Siegel RW, Bizios R. An in vitro evaluation of nanophase alumina for orthopaedic/dental applications, Bioceramics 11: Proceedings of the eleventh International Symposium on Ceramics in Medicine, 5 November 1998. New York, NY: World Scientific, New York; 1998.

22. raybiotech.com [homepage on the Internet]. Norcross: RayBiotech, Inc.; 2014. Available from: http://www.raybiotech.com/. Accessed November 1, 2013.
International Journal of Nanomedicine

\section{Publish your work in this journal}

The International Journal of Nanomedicine is an international, peerreviewed journal focusing on the application of nanotechnology in diagnostics, therapeutics, and drug delivery systems throughout the biomedical field. This journal is indexed on PubMed Central, MedLine, CAS, SciSearch ${ }^{\circledR}$, Current Contents ${ }^{\circledR} /$ Clinical Medicine,

\section{Dovepress}

Journal Citation Reports/Science Edition, EMBase, Scopus and the Elsevier Bibliographic databases. The manuscript management system is completely online and includes a very quick and fair peer-review system, which is all easy to use. Visit http://www.dovepress.com/ testimonials.php to read real quotes from published authors. 